\title{
Botball Kit for Teaching Engineering Computing
}

\author{
David P. Miller \\ School of AME \\ University of Oklahoma \\ Norman, OK 73019
}

\author{
Charles Winton \\ Department of CIS \\ University of N. Florida \\ Jacksonville, FL 32224
}

\begin{abstract}
Many engineering classes can benefit from hands on use of robots. The KISS Institute Botball kit has found use in many classes at a number of universities. This paper outlines how the kit is used in a few of these different classes at a couple of different universities. This paper also introduces the Collegiate Botball Challenge, and how it can be used as a class project.
\end{abstract}

\section{Introduction}

Introductory engineering courses are used to teach general principles while introducing the students to all of the engineering disciplines. Robotics, as a multi-disciplinary application can be an ideal subject for projects that stress the different engineering fields. A major consideration in establishing a robotics course emphasizing mobile robots is the type of hands-on laboratory experience that will be incorporated into the course of instruction. Most electrical engineering schools lack the machine shops and expertise needed to create the mechanical aspects of a robot system. Most mechanical engineering schools lack the electronics labs and expertise needed for the actuation, sensing and computational aspects required to support robotics work. The situation is even more dire for most computer science schools.

Computer science departments typically do not have the support culture for the kind of laboratories that are more typically associated with engineering programs. On the other hand, it is recognized that computer science students need courses which provide closer to real world experiences via representative hands-on exercises. This need is usually addressed in the context of software, but it also pertains to topics more closely linked to physical hardware, which certainly characterizes robotics.

What is needed is a robot prototyping system that requires a minimum of support infrastructure, but has the depth and flexibility to allow serious engineering to take place. The Botball kit was originally designed for use in high schools, and requires no tools except for a computer and an occasional application of hot glue to speed assembly. The kit is being used in over thirty colleges and universities in at least four countries. The remainder of the paper describes the kit and how it is being used in a couple of different schools in different disciplines to teach different aspects of engineering. We will also describe some of the motivations both for using this kit and for having students work on robotic projects as motivating and educational factor. 


\section{The Botball Kit}

The Botball kit has been a work in progress since the early 90's when KISS Institute for Practical Robotics (KIPR), a non-profit organization focusing on learning and skills development through the application of technology, particularly robotics, was founded. In its current form, the kit includes the Handy Board [14] (with charger and serial interface), LEGO RCX (with serial interface), DC motors (both LEGO and modified servo motors), servo motors, touch sensors (3 types), light sensors (general, break beam, and reflectance), proximity sensors (both optical and SONAR), a modified CMUcam and over 1900 pieces of LEGO, mostly LEGO Technic. Cross compatibility between the Handy Board and RCX environment is maintained where reasonable (e.g., at the hardware level, the DC motors, whether LEGO or modified servos, work for both environments). Programming is accomplished via a host PC using Interactive C, 4.3 which is included on the support CD.

Interactive C, which originated with the MIT 6.270 Contest extends C syntax to include (limited) capability for initiating and managing concurrent processes, thus facilitating implementation of robotic paradigms such as subsumption. Technically, IC compiles to P-code. The IC environment manages the interface between $\mathrm{PC}$ and processor board, including download of firmware (the P-code interpreter). Sensors and ports on the processor board are usually controlled via $\mathrm{C}$ library functions supporting the processor board being used, the executables for which are automatically downloaded to the processor board by the IC environment at initialization. Interactive C (IC 4.3), which is the property of KIPR, is offered as freeware through the KIPR web site. IC 4.3 supports both the Handy Board and RCX, and can be run from any one of MS Windows (W95 on), MAC OSX (and earlier MACs), and Linux.

The Botball kit has evolved over time, with earlier versions used for robot building labs at the annual AAAI convention. The current version is employed for the KIPR Botball Educational Robotics Program for Middle and High Schools, which had its inception in 1997 [3]. The kit is currently available via KIPR's web site store.

It also needs to be noted that the Handy Board distributed with the Botball kit is an extension of the original MIT design, incorporating an expansion board to facilitate the use of servo motors and for sending digital signals to external devices. Other minor modifications have been made to the board to facilitate the use of optical range sensors.

Replacements are available from KIPR for all kit parts, a major plus for lab sustainability.

\subsection{FREEWARE}

There are two significant pieces of freeware that support the Botball kit, Interactive $\mathrm{C}$ and MLCAD.

\subsubsection{Interactive $C$ (version 4.3)}

The primary piece of freeware employed for the course is Interactive $\mathrm{C}$, available from the KIPR web site [5]. The interface for IC 4.3 is a recent creation of Randy Sargent, who continues to be the primary developer for IC. 
It is significant that the same interface is used for both the Handy Board and the RCX, which employ different microprocessors.

The interface window utilizes tabbed frames, one of which is an interaction window for directly executing functions on the processor board. A tabbed frame is created for each new source file the user initiates or loads. Source code is automatically indented on load or on entry. The interface provides very thorough on-line documentation, and reasonable source code entry capabilities, including colorizing of text according to category. A suitably large font for class display is among the options. An upload capability is provided for capturing global data arrays generated during robot operation. The data capture is in text format with options for direct import into an analysis tool such as Excel. Pictorial instructions facilitate establishing a working link between PC and processor board, but debugging support otherwise is minimal. At present only a serial interface is supported, which may require purchase of a USB to serial adapter for newer PCs. A processor board simulator is in the offing.

\subsubsection{MLCAD}

The second piece of freeware is MLC AD [11], a LEGO CAD package constructed on top of a freeware vector graphics package called LDRAW. MLCAD includes renditions of almost all LEGO parts that have ever been manufactured. In some respects the package is rough around the edges, but it is quite effective for documenting constructions and producing step by step renditions of constructions. As an example, a fundamental demobot base unit constructed from LEGO Technic as rendered in MLCAD is shown in Figure 1.

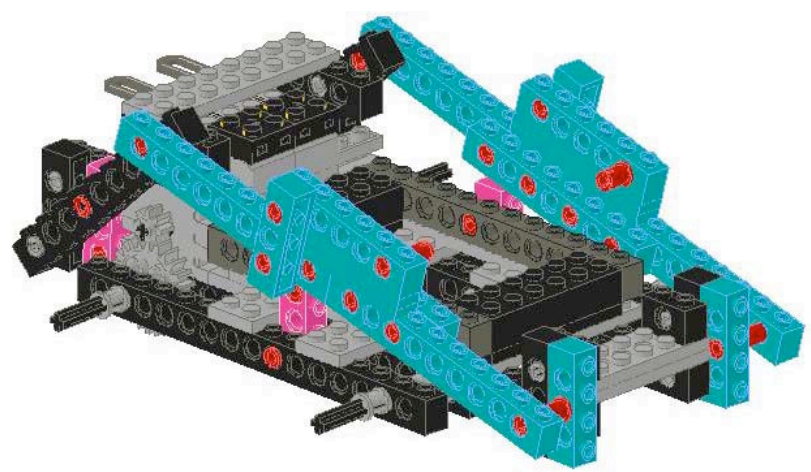

Figure 1. Demobot base unit

MLCAD also provides parts lists for constructions; e.g.,

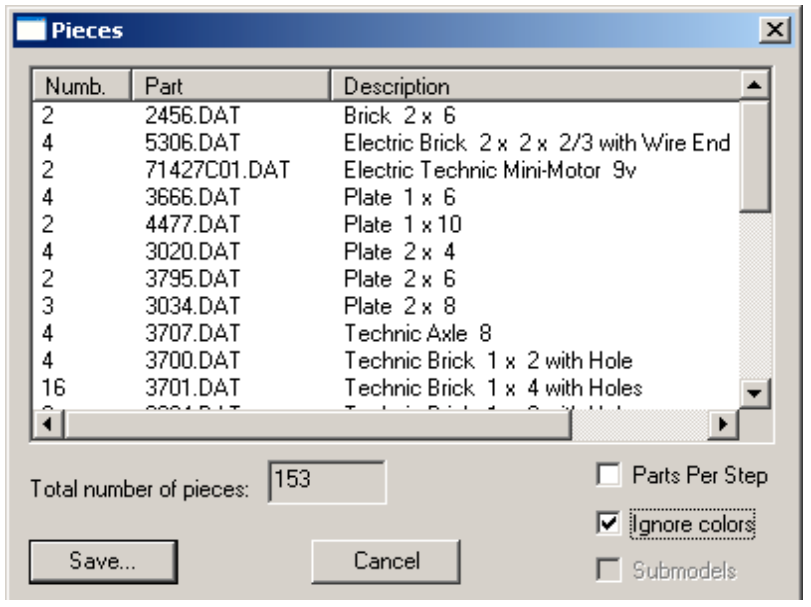


User-defined models can be also be employed as parts in larger models. For example, if the base unit in Figure 1 is added to the MLCAD parts list, then it is a small matter to add treads or wheels from within MLCAD to obtain constructions as illustrated in Figure 3.

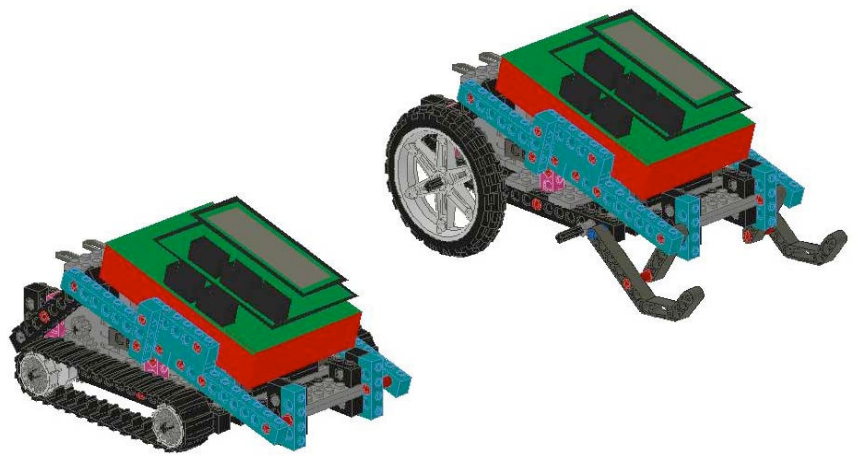

Figure 3. Demobot variations with Handy Board

\section{Robots for Introduction to Engineering}

\subsection{Overview}

For the past three years at the University of Oklahoma, we have merged the Engineering Computing and Introduction to Engineering courses in some sections. These merged courses have used a version of the Botball robot kit as the major tool for teaching computer skills, the design process, project organization, and general engineering techniques. The class meets in two 2-hour sessions per week allowing adequate time for both lecture and in-class hands-on work.

This course is aimed at freshman engineering students and is supposed to cover several objectives:

- Overview of the major engineering disciplines

- Introduction to the engineering design process

- Engineering ethics

- Basic engineering productivity and analysis tools

- Introduction to computer programming

We have successfully used design projects around robots, using the Botball kit, to meet all of these educational objectives

\subsection{Structure of the Course}

Two basic course outlines have been used for this class over the past four years. The first was based on the Managing Creativity curriculum [10].

\subsubsection{The Managing Creativity Approach}

In this version of the class the students are broken into groups of about a dozen to form "companies". They are then tasked to develop a product idea, form engineering groups to develop various portions of the product and then prototype the system. 
Throughout this process the instructor presents tutorials on a variety of topics including:

- Programming in $\mathrm{C}$ using IC4

- Personality categorization (e.g., Keirsey—Bates personality types [4])

- Engineering topics and specializations

This version of the course spent a lot of the time on the management and conflict resolution issues. Using large groups, and the relatively small amount of pieces, processors, etc available in a single Botball kit forced the group to spend a lot of time on organizational issues. They had to decide on a product idea that could be prototyped with the kit. The more difficult task was to come to agreement on what that product idea would be.

A typical example of a project that would arise from the class was an automated party photographer. This was a robot that would wander around a room, and when it got near a person, it would back off a bit and tape a snapshot. The prototyped version from the Intro to Engineering class was somewhat limited in that the robot constructed simply wandered in what it hoped wan an unobstructed plain with the occasional person - in other words, it would photograph anything it noticed sticking out of the floor. Students in the class did feel vindicated in their basic product idea when the next semester it was discovered that professors at Washington University had come up with the same concept and (mistakenly) claimed that they had produced the first robot photographer [9].

\subsubsection{The Multi-Project Approach}

In the second version of the course the students were broken into groups of three for all of their projects. The memberships of the groups changed throughout the semester. On the first project the teams were selected based on the instructor's choice to try and spread people around so that every group had someone with programming experience and that students who had expressed interest in the different fields of engineering were mixed up, rather than putting all of one type together in a single group.

In the second project each student made a list of the top five people they wished to work with. The groups were formed to try and match as many of those requests as possible.

For the final project, there were four different projects to choose from, and different roles for each of the projects. The students used an online bidding and registration system to apply for the project and position they wanted. They could also view else had received assignments, and then relinquish their position and apply for another if they wished. After a day, all the positions were locked into place.

Throughout the term, three major projects were given where complete robot systems must be designed, built and programmed. The first project was a relay race where a series of two robots race between two stacks of soda cans and around a central obstacle, trying to make the best time without knocking over any cans. A marked parabola between the stacks allows some robots to navigate by line following - and a tutorial on line following is given in the course. However other robots manage to follow a straight path by going over the obstacle, and in a few cases picking up and moving the obstacle out of the way (allowing the second robot to go much faster).

The second project required traversing rough terrain as the robot must go over a simulated Moonscape trying to find the leaky habitat (marked with a beacon) and plug the hole, literally, 
with a cork. The terrain used for this project is reconfigurable in three different ways making it (the instructors thought) very difficult to have the robots simply execute a preprogrammed path to get to the habitat. However, it turns out that many of the groups were able to program in all of the paths, and then have the robot, early in its traverse, scan for a specific pattern of rocks to determine which configuration was in play.

The third project, attempted to be substantially different that the previous two. Towards that end the students were given a choice of four possible projects, working in larger groups, to prototype a project (much in the spirit of the Managing Creativity approach). The four options for this past year were: MySDI - the personal security robot; Chembot - an automated system for measuring out and mixing liquid chemicals; Y-prize - long distance robot racer; and Servbot - the personal caterer. The exact definition of each of these was largely left up to the team, and in this project significant augmentation of the kit was allowed.

In addition to the projects, additional assignments are given throughout the term that tie programming to other computer tools needed by engineers. For example, programs are written to store sensor values in an array. The arrays are then uploaded from the robot to the PC and placed into spreadsheet programs for analysis and graphing. This exercise teaches rudimentary data structures as well as spreadsheet techniques.

The third project was most educational - for the instructors. One of the important things we learned was that doing a project with a large group in a short time really shows whether or not you have adequately taught the team management and conflict resolution strategies to the students (we had not). But despite some of the personnel difficulties, the majority of the projects resulted in creative, and for the most part successful robots, and enthusiastic students.

\section{An AI Robotics Course}

At the University of North Florida, the Botball kit has been used for the past 2 years to support a course in AI robotics offered by the Department of Computer Science. Cross fertilization with ME occurs at the graduate level with exploration of mobile robots interacting with stationary robot manipulators. The term AI robotics (Murphy [12]) is used to emphasize robotics that focuses on mobile robots operating under conditions of uncertainty. The Botball kit provides an ideal means for establishing and maintaining an effective support laboratory for an upper level major elective in AI robotics, providing hands-on experience with concepts covered by the course. The student audience is well-prepared in systems software, so the focus is on AI concepts and the means to achieve working models that utilize the concepts. Students from engineering as well as computer science have taken the course. As is perhaps appropriate for computer science departments, the approach employed does not require dedication of a room for an instructional lab.

The Botball kit developed by KIPR provides a comprehensive solution for supporting AI robotics courses, providing all of the advantages of using a component assembly approach. Since the Botball kit incorporates both the Handy Board and RCX, work done by others utilizing either of these technologies (e.g., the successful Handy Board courses described in [1,2,7 and 8] or the RCX course described in [6]) can be easily transferred. For work done with prefabricated base units, it is a generally possible to produce acceptable counterparts using the Botball kit to illustrate the same concepts (eg., a sonar ring for avoid behaviors). 
A strong motivator for instituting the course and lab in the manner chosen was the increasing presence of alumnae from KIPR's Botball Educational Robotics Program for Middle and High Schools. The presence of these students was a direct outcome of serving as the host institution for a Botball regional (beginning in 1997). These students and their friends were increasingly requesting an AI robotics course opportunity that reflected the kind of activities characterizing Botball; namely, a course emphasizing autonomous robots, employing AI techniques to achieve both performance and survivability objectives.

The basic course outline is as follows:

- Mobile robots and robotic paradigms, emergent behavior

- The lab and Handy Board - line following using variations of 2 wheel steering (tank treads, wheels of different sizes)

- Touch and light sensors, position sensing devices - combined sensors to seek and turn off lights

- Braitenberg vehicles, reactive paradigm - sonar for obstacle avoidance, multiplexed sonar

- Subsumption, encoders - maze following

- Hybrid paradigms, sensor fusion, DC motors, gears, servos

- Navigation - school figures

- Other propulsion mechanisms, pneumatics - term project

- Camera, detecting color blobs

- Hybrids, cooperating robots, agents

- Mapping

The class is organized in teams of 2 . There are 4 major laboratory exercises and a term project. Each lab involves deployment of a robot model that may be derived from a base design such as the one given in Figure 1 above, or one independently designed by the team. The progression of the labs requires deployment of an increasing amount of sensor technology to achieve lab objectives, reinforcing ideas introduced in lecture and laying a basis for exploring later topics, such as sensor fusion. On the day each lab is due, the teams compete for best performance, which has some bearing on grade (there is an expected minimal performance level). Class follow up sessions examine solutions using different AI robotics paradigms. Shortly after midterm, the specification for the term project is released, which is cast in the form of a game taken from KIPR's Collegiate Botball Challenge. At the end of the term each team must demonstrate performance by playing the game with no opponent (this establishes a relative ranking of teams), then participate in a double-elimination tournament as a test of survivability. A team's entry may consist of more than one robot. All labs require submission of a lab report in a prescribed format, to include a verification of testing outcomes by one of the opposing teams.

\section{Aerospace Engineering Robotics Lab}

In the Spring of 2004, the OU added a new robotics lab to its aerospace curriculum. The goal of this course is to familiarize aerospace students with the electronics and programming now common in various aspects of avionics and space robotics. This senior level course may be the first course involving programming that many of the students have had since their freshman year. 
This course is using the Botball kits in a manner somewhat different to that outlined above. The course first concentrates on sensors - including the CMUcam color tracker. In this module all of the sensors are examined and their output over a range of inputs is plotted against one another (in particular against the sonar which is already calibrated to metric units). Even the camera is calibrated to find out the size of objects at different distances, and then back calculate the focal length of the lens.

The second module in the course concentrates on motors and servos. The students implement encoders and then use the gear motors under bang-bang control and compare their positional accuracy to that of the servos provided in the kit.

The third unit introduces more complex control systems: p-loops, and pd-loops and compares their performance to the positional servo motors. The students create a mobile robot that then performs some positional and speed servoing using the encoders to close the loop. Again the results are analyzed and written up in a report.

The fourth module combines the results of the first three to produce a mobile robot capable of controlled movement. The students create robots that can perform line following and analyze the change in speed verses maneuverability as the rate of turn and sampling rate are changed.

Similar exercises are done with light tracking and following a colored object.

The fifth module covers kinematics and manipulation. A simple 2-DoF arm is built out of the kit materials and inverse kinematics are calculated. Experiments with positional control of the arm are performed and the results written up.

The last portion of the class then tries to combine all of the pieces that have been created so far, into a single unified challenge. The task involves object tracking, dead reckoning, position estimation, manipulation and a large design element. For this final portion we are using the 2004 Collegiate Botball Challenge [13].

\section{The Collegiate Botball Challenge as a Way to Keep Students Programming}

College Botball was originally created to answer the request of Botball alumni students who had participated in the robotics contest as high school students. The annual challenge which now takes place as part of the National Conference on Educational Robotics (co-located in 2004 with the National Conference on Artificial Intelligence) is similar but distinctly different from the high school contest.

The motivation for having a different challenge is two-fold: first, these are college students with potentially more time and access to more expertise than your typical high school student, there fore they should be able to handle a more difficult challenge; second, by having a different contest, it makes it easier to avoid spontaneous challenges from the middle and high school teams who also attend the event. In the past, those challenges had sometimes had embarrassing outcomes for the college teams involved. 
The high school Botball challenge usually has a part of the scoring that can be achieved by having the robot simply run into certain things. In the college version, the game is designed so that all scoring should be the result of intentional manipulation. The college game also relies more heavily on elements of the Botball kit that are often under utilized by the high school teams (e.g., the color camera).

At OU we are offering some travel grants to college students who demonstrate a robot team that can perform in the college challenge. Students wanting to apply for the grants have to assemble, program and demonstrate an entry that can score what is estimated to be a competitive score in the seeding/performance rounds of the college challenge. The top teams from that then do a head to head double elimination tournament to find the winners of the travel grants.

Our hope is that the skills used for designing and programming the robots will take on a new level of importance, and be less likely to fade away as soon as the semester ends.

\section{Conclusions}

End of course student surveys for all of these courses have shown an overwhelming positive response to the robot kits and robot projects. The complaints that were recorded almost all referred to the team interactions - and these problems were much more prevalent with the larger teams ( 4 to 6 people) than in the two and three person teams. Students describe the courses as time intensive, with conceptual content on par with other courses in the curriculum. In the course surveys, the students also have endorsed the competition format employed with lab projects

In providing a comprehensive kit and in serving as a single source of supply, KIPR has provided a sole-source, reasonably priced means for utilizing a Handy Board component-based approach for the support lab for AI robotics. The incorporation of LEGO RCX elements into the kit also permits an instructor to draw on resources already developed for use with the LEGO Mindstorm kit.

Because of the motivation that the robots provide the students, we are able to teach computing techniques far beyond that which would normally be covered by an engineering computing course. These include issues such as: multi-threading, structures, and semaphores. Topics that are more typical of an intermediate computer science curriculum. Computer science students also get advantages from robotics classes. They get to learn aspects of control, mechanics and electronics not addressed in the normal CS curriculum.

In many of the engineering disciplines, students may never have to program during their undergraduate career with the exception of their intro programming course. However, the inclusion of a Botball collegiate game at the National Conference on Educational Robotics, now encourages the students to keep their computer skills active as they prepare for the Summer tournament. We believe that the use of robots as a teaching tool early in the engineering curriculum will both improve computer skills for non-CS engineers, and will also increase the number of students interested in pursuing computer science. 


\section{Course Resources}

Syllabi and additional information on the AI Robotics lab offered at the University of North Florida can be found at:

http://www.unf.edu/ccec/cis/cwinton/html/roboticsclass/f03/roboticsmenu.html

More information about the introduction to engineering and aerospace robotics lab courses can be found at:

http://coecs.ou.edu/David.P.Miller/robot-courses

\section{References}

[1] Beer, R.D., H.J. Chiel and R.F. Drushel, "Using Autonomous Robotics to Teach Science and Engineering, Communications of the ACM 42, (June, 1999), pp. 85-92.

[2] Beer et al at Case Western Reserve.

http://www.eecs.cwru.edu/courses/lego375/

[3] Botball Educational Robotics for Middle and High Schools. http://www.botball.org

[4] Keirsey, David and Marilyn Bates, Please Understand Me: Character \& Temperament Types, Prometheus Nemesis Book Co; 5th edition, 1984.

[5] KISS Institute for Practical Robotics. http://www.kipr.org

[6] Klassner, F., “A Case Study of LEGO Mindstorms Suitability for Artificial Intelligence and Robotics Courses at the College Level", Proceedings of the $33^{\text {rd }}$ SIGCSE Technical Symposium 34,1 (2002), pp. 8-12.

[7] Kumar, Deepak and Lisa Meeden, "A Robot Laboratory for Teaching Artificial Intelligence", Proceedings of the $29^{\text {th }}$ SIGCSE Technical Symposium 30,1 (1998), pp. 341344.

[8] Kumar and Meeden's on-line material. http://mainline.brynmawr.edu/Robots/ResourceKit/

[9] Lewis the robot eyes future in wedding photography http://newsinfo.wustl.edu/news/page/normal/190.html

[10] Managing Creativity-Donna Shirley. http://www.managingcreativity.com/

[11] MLCAD. http://www.lm-software.com/mlcad/

[12] Murphy, Robin, Introduction to AI Robotics, MIT Press, 2000.

[13] The Collegiate Botball Challenge http://www.botball.org/tournament/collegiate.html

[14] The Handy Board. http://www.handyboard.com 
DAVID P. MILLER

Dr. Miller currently serves as the Wilkonson Chair Professor of Engineering in the School of Aerospace and Mechanical Engineering at the University of Oklahoma. His research interests include two main areas: robotics technology and robotics as a mechanism for technology education. His interests in robotics technology are in automated planning, robotics, and communications with automated systems, especially as applied to planetary exploration and assistive technologies. His education interests include robotics for exploring design and as a testbed for the structuring of engineering teams. The kits used in the OU courses were purchased as part of a grant from the Department of Education \# R215K010122.

\section{CHARLES N. WINTON}

Dr. Winton currently is Professor and Graduate Director of Computer and Information Sciences at the University of North Florida. His research interests include AI robotics, cognition modeling and simulation, and medical systems simulation. He has served as coordinator for the Florida Region Botball Educational Robotics Program for Middle and High Schools since 1997. The course at UNF reported in this paper was supported by NSF grant DUE-0126676 (CCLI program). 